\title{
Comparative Analysis of the Antioxidant Response to Salt Stress in Inula crithmoides and Dittrichia viscosa
}

\author{
Juliana CHAURA ${ }^{1,3}$, Mohamad AL HASSAN ${ }^{1}$, Enrico DANISO ${ }^{1,4}$, Oscar VICENTE ${ }^{1}$ and Monica BOSCAIU ${ }^{2 *}$ \\ ${ }^{1}$ Institute of Plant Molecular and Cellular Biology (IBMCP, UPV-CSIC), and ${ }^{2}$ Mediterranean Agroforestal \\ Institute (IAM, UPV). Universitat Politècnica de València, Spain. \\ ${ }^{3}$ permanent address: Department of Biological Sciences, Universidad ICESI, Cali, Colombia \\ ${ }^{4}$ permanent address: Dipartimento di Scienze Agrarie ed Ambientali, Universita degli Studi di \\ Udine, Italy \\ *)corresponding author, e-mail: mobosnea@eaf.upv.es
}

BulletinUASVM Horticulture 72(2) / 2015

Print ISSN 1843-5254, Electronic ISSN 1843-5394

DOI:10.15835/buasvmcn-hort:11490

\begin{abstract}
Salt stress, as well as other abiotic stresses, induces the activation of antioxidant systems in plants, since they cause oxidative stress as a secondary effect. We have analysed the antioxidant response to salt treatments of two related species, Inula crithmoides and Dittrichia viscosa, in terms of accumulation of total phenolic compounds and flavonoids. The most salt-tolerant species, I. crithmoides, was less affected than D. viscosa by oxidative stress in the presence of high $\mathrm{NaCl}$ concentrations, as revealed by its relatively lower contents of malondialdehyde, a reliable oxidative stress biomarker. Consequently, salt-induced accumulation of antioxidant phenolics and flavonoids was much lower in I. crithomoides, as compared to D. viscosa. From these data, we concluded that the higher salt tolerance of I. crithomoides cannot be based in the activation of stronger antioxidant systems, but must be due to other stress responses - such as the control of water and ion transport and the synthesis of osmolytes for cellular osmotic balance.
\end{abstract}

Keywords: Antioxidants, flavonoids, malondialdehyde, phenolic compounds, salt stress.

\section{Introduction}

Many environmental stress conditions, including high soil salinity, cause oxidative stress in plants, to which they respond by activating different antioxidant systems, both enzymatic and non-enzymatic. Among the latter, phenolic compounds, in general, and especially flavonoids have been proposed to play a fundamental role in the mechanisms of abiotic stress tolerance in plants. Yet, the evidence in favour of this hypothesis is mostly based on work carried out with a limited number of model species, as it is far from clear that it can be generalised. Comparative analyses of salt stress responses in genetically related taxa differing in their degree of tolerance may help to establish the relevance and the relative contribution of antioxidants (and other stress responses) to salt tolerance in different species, an aspect that remains largely unknown.

\section{Aims and objectives}

To lend support to the aforementioned ideas, we have correlated the relative salt tolerance of two related species, Inula crithomoides and Dittrichia viscosa (formerly Inula viscosa) - established from the degree of salt-induced growth inhibition - with the level of oxidative stress and the antioxidant contents (total phenolics and flavonoids) in salttreated plants.

\section{Materials and methods}

I. crithomoides and D. viscosa plants, obtained by seed germination and grown in the greenhouse, were treated with increasing salt concentrations $(0,150,300,450$ and $600 \mathrm{mM} \mathrm{NaCl})$ for six weeks. Then growth parameters (fresh weight, water content) were measured and the levels of malondialdehyde (MDA) and the contents of total phenolic compounds and flavonoids, were 

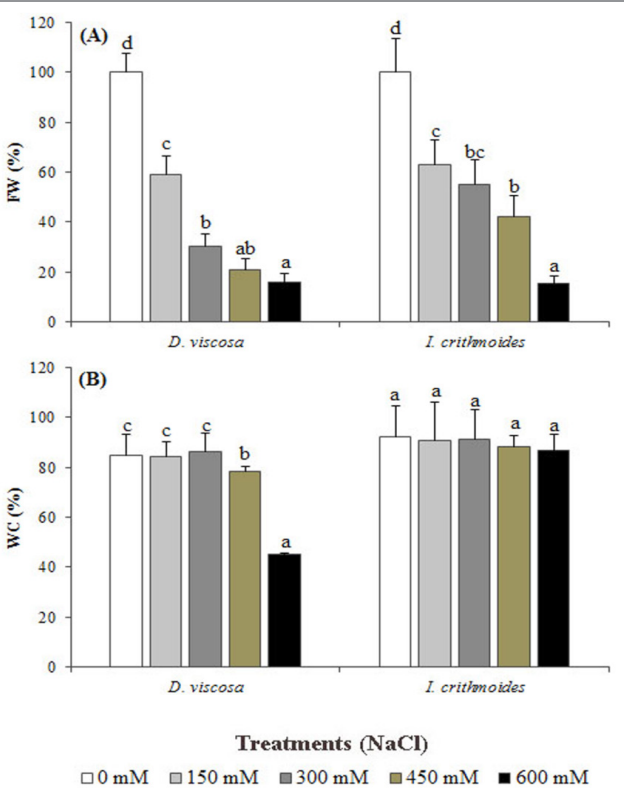

Fig. 1 Growth parameters in D. viscosa and $I$. crithmoides after 6 weeks of treatment with the indicated salt concentrations. (A) fresh weight of the aerial part of the plants, in percentage (\%); the weight of the control, non-treated plants was considered as $100 \%$ for each species. (B) water content percentages WC (\%). Values shown are the means $( \pm S D)$ of five individual plants per treatment $(n=5)$. Different lowercase letters within each species indicate statistically significant differences between treatments, according to Tukey test $(\alpha=0.05)$.

determined in the aerial parts of the plants, using spectrophotometric assays.

\section{Results and Discussion}

Although $\mathrm{NaCl}$ inhibited plant growth in both species, the effect was relatively weaker in $I$. crithomoides, which showed less reduction in fresh weight with increasing $\mathrm{NaCl}$ concentrations (Fig. 1A). This result indicated that I. crithomoides is the most salt tolerant of the two species, in agreement with their distribution in nature; being succulent, it was also more resistant than $D$. viscosa to saltinduced dehydration (Fig. 1B). Despite these differences, it should be pointed out that both taxa are quite resistant to salinity, surviving for six weeks in the presence of high salt concentrations (up to $600 \mathrm{mM} \mathrm{NaCl}$ ).

Malondialdehyde (MDA) is a product of membrane lipid peroxidation and a reliable marker of oxidative stress. Its levels increased in

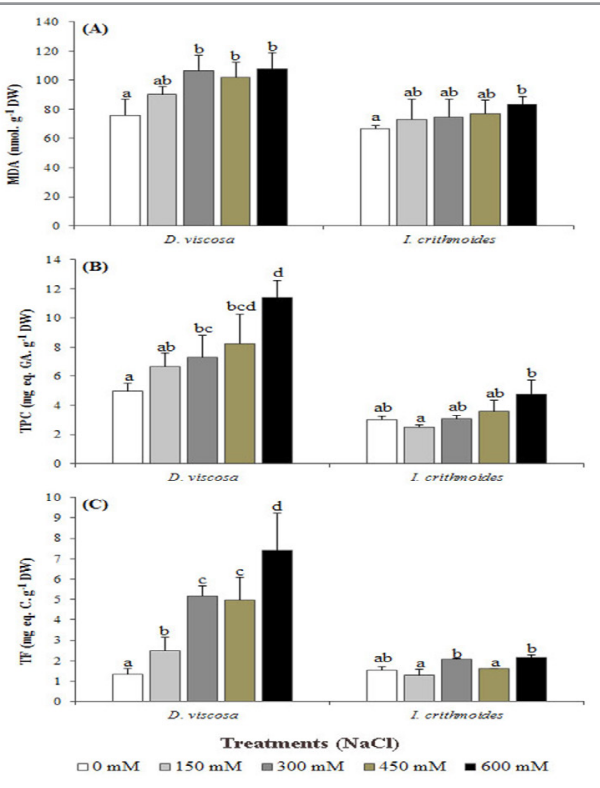

Fig. 2 Oxidative stress marker and non-enzymatic antioxidant levels in D. viscosa and I. crithmoides after 6 weeks of treatment with the indicated salt concentrations. (A) malondialdehyde (MDA). (B) total phenolic compounds (TPC), expressed as 'mg equivalent of gallic acid (GA) per g of dry weight (DW).

(C) total flavonoids (TF), expressed as 'mg equivalent of catechin (C) per g of dry weight (DW). Values shown are the means $( \pm$ SD) of five individual plants per treatment $(n=5)$. Different lowercase letters within each species indicate statistically significant differences between treatments, according to Tukey test $(\alpha=0.05)$.

D. viscosa in parallel with increasing external salt concentration, whereas in I. crithomoides MDA contents were lower and a significant difference with the control plants was only observed in the presence of $600 \mathrm{mM} \mathrm{NaCl}$ (Fig. 2A). Accordingly, salt-dependent accumulation of antioxidants, total phenolics (Fig. 2B) and flavonoids (Fig, 2C) was higher in D. viscosa than in I. crithomoides, the latter showing a significant increase in their levels only at the highest $\mathrm{NaCl}$ concentration tested.

\section{Conclusion}

These results suggest that the higher salt tolerance of I. crithomoides, as compared to D. viscosa, cannot be due to a stronger activation of antioxidant systems, but must be based instead on other stress responses - such as the control of ion transport and the synthesis of specific osmolytes to maintain cellular osmotic balance. 